\title{
Bringing the Farming Community Into the Internet Age: A Case Study
}

\author{
A. B. Deraman \\ Universiti Kebangsaan Malaysia
}

\author{
A. K. Shamsul Bahar \\ Bionergy Sdn. Bhd.
}

a.d@pkrisc.cc.ukm.my

\begin{abstract}
Despite the rapid Internet evolution in Malaysia, the farming community is not getting its full benefit due to many factors. Efforts to get the community use the Internet without the availability of proper resources and appropriate content are ineffective. TaniNet is an interactive on-line agricultural and biotechnological web site aimed at providing the agricultural community with information on the advances of planting materials and practices. The major goal of TaniNet is to get the rural farming community to use the Internet as means of accessing and sharing information and farming practices in Malaysia. In this paper we present the experience of developing and implementing TaniNet and the lessons learned.
\end{abstract}

Keywords: Farming community, informed community, agricultural web site, agricultural portal, incremental development, Internet, software engine.

\section{Introduction}

Focus on the Malaysian Agricultural sector was renewed following the Malaysia economic crisis in 1998 (Shariffaden, 2000). The decline in the exchange rate of Malaysian Ringgit (RM) compared to major world currencies uncovered the vulnerability of the Malaysian food supply and at the same time increased the awareness of the importance of the local agricultural sector.

The shift in exchange rate has caused a dramatic increase in Malaysia's foreign food bill. In 1997, food trade, excluding palm oil, experienced a deficit of RM4.74 billion or $49 \%$ of the total deficit in goods and services. It is also expected that the current exchange rate (RM3.8 = US\$1) alone will increase this value by $30 \%$ (NAP3, 1999).

Due to this, the Malaysian government has placed emphasize on the local agricultural sector. Several issues and challenges have also been identified to help tackle the problem of foreign food dependency and these include (NAP3, 1999):

- The need to ensure adequate supply of safe, nutritious and high quality food at affordable prices.

Material published as part of this journal, either on-line or in print, is copyrighted by the publisher of Informing Science. Permission to make digital or paper copy of part or all of these works for personal or classroom use is granted without fee provided that the copies are not made or distributed for profit or commercial advantage AND that copies 1) bear this notice in full and 2) give the full citation on the first page. It is permissible to abstract these works so long as credit is given. To copy in all other cases or to republish or to post on a server or to redistribute to lists requires specific permission and payment of a fee. Contact Editor@inform.nu to request redistribution permission.
- The need to reduce agricultural labor requirements.

- The need to maximize land utilization

- The need to strengthen the competitiveness of Malaysian agriculture

- The need to enhance sector investment in food production

- The need to transform the smallholders into viable commercial entities

- The need to strengthen industrialization and offshore investment

- The need to ensure sustainable agriculture and forestry development

To ensure that all these challenges are met, the Malaysian Ministry of Agriculture has introduced the Third National Agricultural Policy 1998-2010 (NAP3). The NAP3 aims to meet the national food requirements through the following means (NAP3, 1999):

- Enhancing competitiveness and profitability in agriculture and forestry

- Enhancing the integrated development of the food and industrial crop sub sectors

- Strengthening requisite economic foundation

- Adopting sustainable development

Other than to overcome the challenges through the methods listed, NAP3 also acknowledges the presence of diverse consumer preferences towards products. Thus, the focus of NAP3 includes the use of current conventional as well as the more developed emerging technologies to achieve higher productiv- 
ity and quality and to increase the competitiveness of the sector.

The use of information technology is also emphasized in NAP3. It is hoped that information technology will play an important role in the acquisition and dissemination of new knowledge and technologies to motivate the involvement of youth in the agricultural sector. The utilization of computer simulated scenario analysis also enhances on-farm advice and information exchange to agricultural producers (NAP3, 1999).

In order to achieve the targets outlined in NAP3, the steps need to be taken not only by the government but also by individuals involved in the agricultural sector. An important aspect of any changes is the effective dissemination of information to inform and educate the participating parties (Low, 2000), especially farmers and smallholders. Currently, efforts to inform these target groups are done on individual and small-groups basis and this is not efficient merely because of the rapid development of new beneficial agricultural products due to the advancement in agricultural technology. Hence, a new technological solution is needed to work in parallel with the government efforts to help educate and inform the farmers and smallholders.

Currently, it can be said that there are a number of agricultural resource sites available on the Internet (Di, 2000). Almost all universities that support agricultural science disciplines have agricultural related web sites (Uky, 2000; Iastate, 2000; Usask, 2000). Governmental and world body web sites that are published through their respective Ministry of Agriculture or the like further complement this. Examples include the New Zealand Ministry of Agriculture web site (MAFnet, 2000) and the United Kingdom Ministry of Agriculture, Fisheries and Forestry web site (Maff, 2000) as well as the United Nations Food and Agriculture Organization web site FAO, 2000 ). To add to this, there are privately owned web sites providing valuable agricultural resources and even products (Agnoc, 2000; Aginfonet, 2000). However, most of these sites are tailored for the developed countries or for a specific continent, if not environment.

A major bulk of the information available on the above mentioned web sites are not applicable to the Malaysian agricultural sector due to differences in weather, crops and production techniques. Language barriers also exist as most web sites use English as the medium while most Malaysian farmers are versed only in their mother tongue, the Malay language. An exception to these sites is the Malaysian Ministry of Agriculture website that is available in both languages (Agrolink, 2000).
This paper discusses a case study of the development and implementation of Internet-based project called TaniNet.

TaniNet was created to enable the Malaysian agricultural community to use the Internet as a tool for communication and as a place to share information on agriculture and biotechnology. Indirectly, TaniNet also plays a vital role in attracting Malaysian farmers to use the Internet by providing them with valuable agricultural information online. TaniNet's effort to promote Internet literacy among farmers does not stop at the browser. Efforts have been made to also educate and train the farmers' family members on how to utilize the Internet, or more specifically, TaniNet, to obtain either information on agriculture or solutions to their problems. Several performance indicators, that include page-hit rate, numbers of queries, number of respondents to an online survey and number of personalized services, have measured TaniNet's achievement. Finally, this paper also discusses the lessons learned from the project and its future direction.

\section{Socio-Economic Profile of Farmers}

To assess the economic status and standard of living of the farming community, a survey has been conducted among 607 respondents. This survey provided data on the socio-economic profile of farmers in Selangor (a well-developed state in Malaysia) and the extent to which they have access to information especially using computer facilities. Amongst others, the study highlighted the following findings (TaniNet $2^{\text {nd }}$ Report, 2000):

- Of the total 607 respondent, $81 \%$ were between 20 and 60 years old. $84 \%$ of the total respondents have attained at least primary education. This means that the majority of them were within the labor force age group of 15-64 and literate. Hence they have the ability to acquire new computer skills.

- Farmers received information from a variety of sources: government agencies, mass media, the private sector, farmers' organizations and individuals. Only $70 \%$ of the farmers received adequate agriculture information. Of these, $88 \%$ of the respondents received agriculture information and advice from the Department of Agriculture.

- Farmers are generally interested in using computers to access information. About $15 \%$ of the 607 respondents owned computers and $20 \%$ know how to use computers. Half those who did not own computers were interested to purchase computers.

The study also indicates that farmers in the state of Selangor are gradually moving away from the image of the traditional farmer to that of an entrepreneur farmer. The transformation from traditional farmer to entrepreneur farmer requires external assistance in terms of management skills, accessing acquiring information on various business ventures, and seeking 


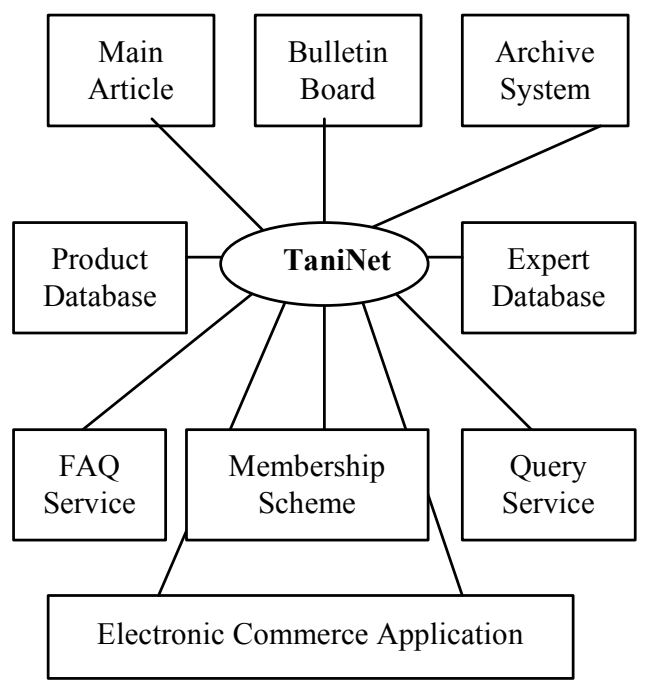

Figure 1: Software engine structure

technical, as well as financial assistance, from agricultural agencies. This can be achieved by way of Internet technology as this technology is available at all times and all places. It is a reliable communication tool in disseminating the information.

\section{TaniNet Project}

TaniNet is essentially an information service toolset. TaniNet started in September 1999 as a project under the Demonstrator Application Grant Scheme or DAGS. DAGS is within the ambit of the National Information Technology Council (NITC). Among the main objectives of this scheme is to introduce the Malaysian rural farming community to agricultural biotechnology through an interactive Internet-based service both in English and the Malaysian native language, Bahasa Melayu. This will include the following specific objectives:

- Provide on-line information and services on agriculture and biotechnology

- Increase local understanding of agricultural biotechnology and local applications of new technology

- Provide a forum for discussion among the rural farming communities through a managed bulletin board and chat room

- Provide searchable information on agricultural biotechnology

- Provide access to expert advice and troubleshooting related to agricultural problems via the Internet

- Educate community as to use of internet to access important and useful information and services

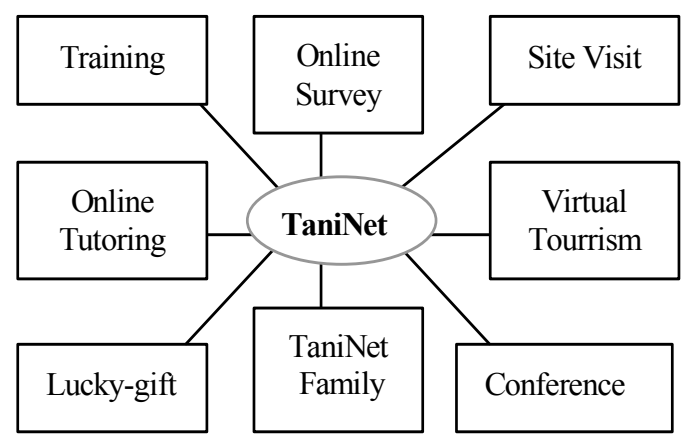

Figure 2: Event-based structure

From an architectural point of view, TaniNet consists of two main structures. One of the structures is a software engine structure as shown in Figure 1. As an ordinary web site, TaniNet is equipped with a typical set of facilities such as articles on agricultural related topics with the support of an archiving system, bulletin board, query and FAQ services and event directory. In order to sustain and maintain a long-term relationship with the targeted community, a membership scheme is also devised within TaniNet.

To the objectives described earlier, TaniNet is designed with product and expert databases. In the long term, these databases will hold up-to-date knowledge on agricultural products and experts available within the agricultural community. Finally, TaniNet is also aimed to provide commercial services in order to self-finance its existence. For this, TaniNet is supported with various applications within e-commerce services.

The other structure is the event-based system. As shown in Figure 2, this structure provides an online survey and site visits activities that are planned periodically to get up-to-date information from the farming community.

Training is also one of the important elements to ensure TaniNet's Information Technology (IT) literacy objective. Scheduled conferences are also parts of the initiatives within TaniNet to bring together the farming community into round table discussions. Online tutoring and virtual tourism are facilities accessible through TaniNet site for education and knowledge advancement. Lucky-gift is a special incentive given to the lucky TaniNet visitor. This mechanism is part of TaniNet promotional schemes. Finally, TaniNet introduces a concept of TaniNet family. This family of the month is chosen from the farming community and published on the web site to encourage others to compete for this special appearance.

\section{Design And Implementation}

At present, the design and implementation of TaniNet is completed as required by the grant. Most of the planned structures 
and components are fully implemented. The following are discussions on components and events:

- Main Article: This component refers to the main article of the TaniNet homepage. It focuses on some agriculturerelated topics of interest. The editor is responsible for determining the topic for a particular edition as well as ensuring sufficient material for that topic. Possible topics include fruits, vegetables and fertilizers. Articles on such topics will commonly contain information on evolution and technological advancements.

- Bulletin Board: The design of the bulletin board is based on an interactive concept on which messages can be posted and replied. Messages posted can be in the form of text, image or even voice. They can also be posted nonelectronically by filling special forms. Each message will be assigned an expiry date and will expire automatically. The bulletin board also consists of three other subcomponents namely advertisements, current events and queries. The query component for example, enables farmers to pose questions regarding agriculture. The relevant experts will answer those questions. The editor can identify these experts from TaniNet's expert database. Farmers may also generate informal discussions on a current topic amongst themselves.

- Online Survey: Surveys provide one way of obtaining information that can be used to improve services offered by TaniNet. They also provide a means for obtaining empirical evidence on certain issues. However, filling in surveys is not something that users enjoy. The number of responses received affects the quality of information that can be extracted. Bearing this in mind, the component is designed in such a way that users are encouraged to participate. As an incentive, gifts are given away to randomly selected survey participants. All information obtained from surveys is confidential and will only be used to improve the level of services provided.

- Membership Scheme: A membership scheme has been devised to maintain a core of committed TaniNet users. Certain services offered by TaniNet are classified members-only in order to attract membership. These include:

- free access to the TaniNet database of articles and other agricultural information;

- participation in TaniNet activities such as organized trips, seminars and conferences;

- personal email address and account with the @ taninet.com.my domain.

There are three categories of membership: student, individual and corporate. Registration can be performed online via the TaniNet web site. Members are registered on a yearly basis and need to renew their membership accordingly.

- Archive System: In each edition of the TaniNet homepage, material such as the main article can be identified for archiving so that they can be referred to in future. The TaniNet archive is a repository for such material. All archive entries are indexed for retrieval purposes. .

- Expert Database: One of the objectives of TaniNet is to function as a bank of experts. Experts in the field of agriculture are identified and appointed as TaniNet affiliates. A database of information about experts in various aspects of agriculture is a great asset to TaniNet. As one of its services, TaniNet can be consulted to assist in identifying experts who could offer advice or services. Such a service is provided commercially as a secure online service.

- Search Engine: The TaniNet homepage incorporates a link to a search engine. The search engine is designed to retrieve information from the current edition of the homepage as well as from the archive system described above.

All TaniNet software components are put into a web server. Those components accessible by the public as well as TaniNet members are accessed via the TaniNet homepage. Components for administrative tasks used by the management staff such as the editor are accessed separately. An example of such a component is the Query Management System (QMS) that assists in handling and managing incoming queries and composing responses received from experts.

The TaniNet web site (http://www.tanınet.com.my) has been set up in which the TaniNet homepage is published. In an effort to bring the farming community closer to TaniNet, a number of training sessions are provided. A conference was also organized to achieve the objective. The farming community is also brought closer to agriculture suppliers through advertisements placed in the TaniNet web pages.

\section{Community Development}

In applying Internet based applications to community development, the use of local content and culture compatibility has been very much emphasized (Baharudin, 2000; Karim, 2000). With this in mind, TaniNet has been developed using indigenous content ranging from popular style articles to academic and research articles from Malaysian scientists and agriculturalists. The web site presentation is based on a magazine format with updates done regularly, from weekly for announcements and news articles to longer intervals for other components of the website. The user interface has been designed to accommodate both the farmer in the field and the scientist (expert) in the laboratory. 
An important component in the implementation of TaniNet is training for users and potential users. Briefings and talks for users at government agencies and corporations are carried out to introduce TaniNet. This is also to promote use of TaniNet as a tool and platform for community development, both in terms of building commercial communities and for social and economic development. Members of the farming community are given hands-on introductory training on the use of personal computers, accessing the Internet and skills to access information and services offered by TaniNet. The training modules and implementation are tailored to achieve maximum impact within the constraints of time and infrastructure availability. An example to dispel any fear of technology among the rural farmers and the older generation is the disassembling of the microcomputer and letting them hold and touch the components while explaining in simple language the functions of the components. For instance, the computer mouse is disassembled to show its simple internal structure including the way the mouse ball operates. At this phase of TaniNet the beginnings of a mind set change has been observed among the rural farming community. Interestingly, with the demonstration of TaniNet, a mind set change as to the use of IT has also been observed among senior government officials and community leaders.

Community development efforts are carried out with close cooperation of the Farmers Organization Authority at the Federal and State levels, and the local farmers' associations at the district or village levels, namely Area Farmers Associations. For this phase, four Area Farmers Associations in the state of Selangor (one of the states in Malaysia) have been chosen as the initial target population. TaniNet has been well received by the farmers associations and their members judging by their response to the training sessions, feedback from their community leaders and results of the performance indicators.

Among the many comments received is that the TaniNet website provides a friendly user interface, easy and fast to access and download. An attractive feature for the farming community is that they can place advertisements of their products onto the website. Marketing has been a main concern among the small and medium scale farmers and now TaniNet may have provided an answer to their need. The ability to pose queries and questions as to their individual problems and getting responses within 24 hours, at least in the form of acknowledgement, has impressed the farmers as to the capability of IT applications, and TaniNet has provided the farmer easy access to the experts in the respective fields.

\section{Project Management}

The planning and development of TaniNet was started six months before the actual launch of the project itself. It involved six personnel at senior management level with wide experience in corporate business management, information and communication technology as well as agriculture. This team established a predetermined course of action, including policies and procedures to reach the set objectives of TaniNet. The following aspects were used as a basis in planning the project:

- Strategy to be followed to achieve the objectives

- Organizational structure and policies for empowerment

- A budget of expenditure based on manpower, material, machine and overheads

- A time schedule for delivery and processes

- Projections set out with clear and definite milestones

- Clear lines of communication and control

- Standards of performance and methods of measurement The initial plans were revised and reorganized based on feedback as well with reference to other developments. At the initial implementation stage major problems that were encountered were staffing and location as well as logistics. A project manager and three part-time assistants were appointed. It operated from a temporary project office at the IT company premises while the site office was being renovated. Subsequently the IT staff was hired and moved to the site office about one month after the launch. The editorial personnel were working from another location initially and relocated to the site office a few weeks after the IT personnel started full operation.

The project site office was fully functional one and a halfmonth after the launch of the project. The management committee was later empanelled to oversee the development as the project increased in complexity.

Quarterly reports were formulated and published for the perusal of the project committee of the National IT Council and a full audit of operations (editorial and IT) as well as financial was conducted by the project committee from the National IT Council. Plans that were initially developed were closely followed and where possible variances were addressed. The project management team played a critical role in the course of the project.

\section{Performance Evaluation}

Throughout the development and implementation of TaniNet, several attributes have been chosen to represent performance indicators. Examples of these include:

- Hit rates

- Number of on-line survey respondents 
- Number of queries

- Number of membership applications

- Number of business transactions

Performance indicator attributes are used to indicate whether the project is on track towards achieving its primary objectives. Table 1 shows the number of hits based on a specific period.

\begin{tabular}{|l|r|r|}
\hline \multicolumn{1}{|c|}{ Month } & Hit count & $\begin{array}{c}\text { Cumulative } \\
\text { hit count }\end{array}$ \\
\hline $\begin{array}{l}\text { Nov.1999 - Feb. } \\
2000\end{array}$ & 1044 & 1044 \\
\hline March 2000 & 722 & 1766 \\
\hline April 2000 & 794 & 2560 \\
\hline May 2000 & 1335 & 3895 \\
\hline June 2000 & 1773 & 5668 \\
\hline July 2000 & 3643 & 9311 \\
\hline August 2000 & 2457 & 11768 \\
\hline September 2000 & 1887 & 13655 \\
\hline October 2000 & 1892 & 15547 \\
\hline November 2000 & 2327 & 17874 \\
\hline
\end{tabular}

Table 1: Number of TaniNet visitors

A cumulative total of nearly 4000 hits were achieved by the end of May 2000. It is important to note that the TaniNet web site was not widely publicized. With more promotional efforts being made such as in conference and seminar presentations, the number of hits is getting higher. This shows that TaniNet is getting wider coverage and maintaining its popularity among cyber citizens. It is only recently that the website was registered with search engine sites such as

http://www.cari.com.my, tttp://www.catcha.com.my, http://www.yahoo.com and http://www.altavista.com.

Another attribute is the number of on-line survey respondents (TaniNet Survey, 2000). In September 2000, the cumulative number of respondents was 332 . This shows that there is interest within the society as the questions are mostly related to agricultural issues such as where the respondent could consult for agriculture-related problems.

The number of queries posted can also be used to gauge the performance of TaniNet. By November 2000, TaniNet had received 234 queries regarding various agricultural issues (Table 2). One query received regards the problem of Jasmine plants that had been infested with white fly. Another query was about the most suitable fish that eats weeds in ponds. The internal editor, with the help of the experts, prepared answers to the queries.

212

\begin{tabular}{|l|c|}
\hline \multicolumn{1}{|c|}{ Question type } & Query count \\
\hline Cash crop & 19 \\
\hline Soil & 17 \\
\hline Aquaculture & 10 \\
\hline Horticulture & 52 \\
\hline Finance & 8 \\
\hline Agroforestry-tour. & 7 \\
\hline Marketing & 11 \\
\hline Biotechnology & 12 \\
\hline Others & 98 \\
\hline \multicolumn{2}{r|}{ Total } \\
\hline
\end{tabular}

Table 2: Number of queries

The performance of TaniNet has also been evaluated in terms of registered members as shown in Table 3. Although most of the members are local, it is interesting to note that there is also interest from the international community represented by the small number of international members of TaniNet. Most of these are from Singapore, US, Canada, Japan, Brunei and Indonesia.

\begin{tabular}{|c|c|}
\hline Country & Member count \\
\hline Malaysia & 1969 \\
\hline Others & 73 \\
\hline Total & 2042 \\
\hline
\end{tabular}

Table 3: TaniNet membership

While more than 30 companies have already placed their advertisements in TaniNet, only 5 Internet orders have been executed. This indicates the business to customer (B2C) portion of TaniNet was not well accepted.

\section{Lessons Learned}

Towards the end of this one-year project and thus the grant period, the management of TaniNet has begun to explore ways of sustaining and maintaining the web site. The initial idea of generating income from advertisements was not implemented due to the availability of free advertisement space throughout the cyber globe. This lesson has also stopped the management from introducing a fee for the query service. Perhaps in this initial stage of technology cultural transformation, the government has to put more public financing into such projects.

To bring the farmer's family to the technology, focus should be made on the younger generation. As "old habits die hard", it is more difficult to educate the older farmers than their chil- 
dren to adapt to the Internet. In the long run this will prepare the country with potential Internet age farming generations. Nevertheless, it is important to obtain the support of the elders. Involvement of the elders at least at the aware-ness level is crucial for total community development.

It was also learnt that the event-based approach is one of the most effective ways of promoting Internet usage. However, organizing and managing such events require even bigger investment in terms of money and manpower. In this case the "smart partnership" among the government agencies, private sectors and the community will ensure the right governance with strong financial and good management structure.

Another interesting lesson learned is the importance of indigenous content and use of the local language in the web site. In this case for example, local news, events and farm product advertisements should be tailored to the different groups within the community. This will facilitate the management of incremental development through small group participation towards an informed community.

\section{Conclusion}

In this paper, a case study of an Internet-based project called TaniNet, to promote the use of IT within the farming community was presented. With the proposed software engine and event-based structures, TaniNet has become a comprehensive toolset as an interactive and on-line information service provider. TaniNet is also the platform for future communication and to redress the digital divide between the farming community and others. Nevertheless, more effort and time are needed in training and promoting Internet technology within the farming community before significant impact can be seen.

The process of bringing the farming community into the Internet age is continuous in nature, thus the outcome of a short term project such as TaniNet is not sufficient. Ultimately, more comprehensive as well as long-term projects with economic sustainability are needed. Such projects should be integrated within the community activities. As for future direction, the sustainability issue of TaniNet may be addressed by introducing a business-to-business (B2B) platform within the toolset. This platform becomes the enabler for implementing internet-based business process among leading agencies within the farming community. Income generated from the business process can be used to maintain and sustain the TaniNet. At later stages, TaniNet is planned to become a specialized agricultural portal for the agricultural community within Malaysia.

\section{Acknowledgement}

The support from Demonstrator Application Grant Scheme (DAGS) through the National Information Technology Council (NITC) and the assistance of MIMOS is duly acknowledged.

\section{References}

Aginfonet, (2000), "Aginfonet web site". nttp./Www.aginfonet.sk.ca/ (24 April 2000).

Agnoc, (2000), “Agricultural Network Information Center web site". ttp://Www.agnoc.com/ (7 MayTa 2000).

Agrolink, (2000), “Agrolink: The Ministry of Agriculture Malaysia web site". http://agrolink.moa.my/ (24 April 2000).

Baharudin, S. A. (2000), Preservation of Culture in an Internetworked World. R. A. Rahim \& K. J. John (Eds), Access, Empowerment and Governance in the Information Age. Building Knowledge Societies Series, Volume I: NITC (Malaysia) Publ., 68-75.

Di, (2000), "Di’s Agricultural Links web page". http://WwW.ozema1l.com.au/ dkgsoft/agr.htmI 24 April 2000).

FAO, (2000), "Food and Agriculture Organization of the United Nations web site". tttp://www.fao.org/(24 April 2000).

Iastate, (2000), "Iowa State university College of Agriculture web site". ttp://www.ag.1astate.edu/(24 April 2000).

Karim, W. J. (2000), Ethics for Global Civil Society: Non-Westerner Perspectives. R. A. Rahim \& K. J. John (Eds), Access, Empowerment and Governance in the Information Age. Building Knowledge Societies Series, Volume I: NITC (Malaysia) Publ., 53-66.

Low, V. (2000), Education System For Knowledge Societies: Issues \& Assumptions For Reform. R. A. Rahim \& K. J. John (Eds), Access, Empowerment and Governance in the Information Age. Building Knowledge Societies Series, Volume I: NITC (Malaysia) Publ., 7886.

Maff, (2000), "The United Kingdom Ministry of Agriculture, Fisheries and Forestry web site". tttp://www.maff.gov.uk/(28 April 2000).

MAFnet, (2000), "New Zealand Ministry of Agriculture web site". tttp://WwW.mat.gov.nz/MAFnet/(24 April 2000).

NAP3, (1999), The Ministry of Agriculture Malaysia. Third National Agricultural Policy (1998-2010). Ministry of Agriculture Malaysia: Kuala Lumpur. 1999.

Rahim, R. A. et al, (2000), Access and Equity: Benchmark for Progress, Infosoc Malaysia 2000 Proceedings, Organised by NITC (Malaysia), 7-9 June 2000, Kuching Sarawak. 104 pages.

Shariffaden, M. A. (2000), The Changing World: ICT and Governance. R. A. Rahim \& K. J. John (Eds), Access, Empowerment and Gov- 


\section{Farming Community}

ernance in the Information Age. Building Knowledge Societies Series, Volume I: NITC (Malaysia) Publ., 1-12.

TaniNet Survey, (2000), Report of online survey for TaniNet users, DAGS Report, TaniNet Project, UKM-MTDC Bangi, Selangor, Malaysia, May 2000.
TaniNet $2^{\text {nd }}$ Report, (2000), Second Quarter, DAGS Report, TaniNet Project, UKM-MTDC Bangi, Selangor, Malaysia. February 2000.

Uky, (2000), "The College of Agriculture University of Kentucky web site". http://www.ca.uky.edu//24 April 2000).

Usask, (2000), "University of Saskatchewan College of Agriculture". tttp://www.ag.usask.ca/ (26 April 2000). 